INPLASY

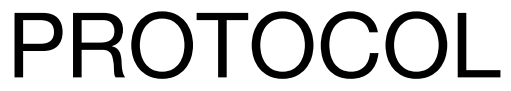

To cite: Zhang et

al.Acupuncture and other related interventions for the treatment of sciatica: Protocol for a scoping review of systematic reviews and metaanalyses. Inplasy protocol 202220118. doi: 10.37766/inplasy2022.2.0118

Received: 27 February 2022

Published: 27 February 2022

Corresponding author: Jia Li

ljlijia@163.com

Author Affiliation: Hubei University of Chinese Medicine.

Support: No.2019XZZX-ZJ006.

Review Stage at time of this submission: Preliminary searches.

Conflicts of interest: None declared.

\section{Acupuncture and other related interventions for the treatment of sciatica: Protocol for a scoping review of systematic reviews and meta-analyses}

\author{
Zhang, Y1; Liang, F2; Liu, S3; Zhang, Y4; Li, J5.
}

Review question / Objective: The objective of this scoping review is to conduct a comprehensive review of the systematic reviews and meta-analyses about acupuncture for the treatment of sciatica and to assess the breadth and methodological quality of them.

Condition being studied: Sciatica is a debilitating condition that is a result of the sciatic nerve or sciatic nerve root pathology. Affected patients experience pain and paresthesias in the sciatic nerve distribution or an associated lumbosacral nerve root. With the development of society and the change of modern lifestyle, the incidence of sciatica has become higher and higher. It not only reduces the quality of life but also incurs consider-able medical expenses. The efficacy of western medicine in the treatment of sciatica is not satisfactory. However, acupuncture, as therapy with a long history in China, has been reported to have significant effects on reducing pain and improving the quality of life among sciatica sufferers. Sciatica is a debilitating condition that is a result of the sciatic nerve or sciatic nerve root pathology. Affected patients experience pain and paresthesias in the sciatic nerve distribution or an associated lumbosacral nerve root. With the development of society and the change of modern lifestyle, the incidence of sciatica has become higher and higher. It not only reduces the quality of life but also incurs consider-able medical expenses. The efficacy of western medicine in the treatment of sciatica is not satisfactory. However, acupuncture, as therapy with a long history in China, has been reported to have significant effects on reducing pain and improving the quality of life among sciatica sufferers.

INPLASY registration number: This protocol was registered with the International Platform of Registered Systematic Review and Meta-Analysis Protocols (INPLASY) on 27 February 2022 and was last updated on 27 February 2022 (registration number INPLASY202220118).

\section{INTRODUCTION}

Review question / Objective: The objective of this scoping review is to conduct a comprehensive review of the systematic reviews and meta-analyses about acupuncture for the treatment of sciatica and to assess the breadth and methodological quality of them. 
Condition being studied: Sciatica is a debilitating condition that is a result of the sciatic nerve or sciatic nerve root pathology. Affected patients experience pain and paresthesias in the sciatic nerve distribution or an associated lumbosacral nerve root. With the development of society and the change of modern lifestyle, the incidence of sciatica has become higher and higher. It not only reduces the quality of life but also incurs consider-able medical expenses. The efficacy of western medicine in the treatment of sciatica is not satisfactory. However, acupuncture, as therapy with a long history in China, has been reported to have significant effects on reducing pain and improving the quality of life among sciatica sufferers. Sciatica is a debilitating condition that is a result of the sciatic nerve or sciatic nerve root pathology. Affected patients experience pain and paresthesias in the sciatic nerve distribution or an associated lumbosacral nerve root. With the development of society and the change of modern lifestyle, the incidence of sciatica has become higher and higher. It not only reduces the quality of life but also incurs consider-able medical expenses. The efficacy of western medicine in the treatment of sciatica is not satisfactory. However, acupuncture, as therapy with a long history in China, has been reported to have significant effects on reducing pain and improving the quality of life among sciatica sufferers.

\section{METHODS}

Participant or population: We will include patients diagnosed with sciatica.

Intervention: Treatment group intervention at least includes a kind of acupuncture therapy (such as acupuncture, electroacupuncture, auricular acupuncture, etc.) or moxibustion therapy.

Comparator: We will include any study that includes comparators.

Study designs to be included: Include all meta-analysis and systematic review on acupuncture for sciatica.
Eligibility criteria: 1) Publications were not full reports;(2) Protocol of reviews.

Information sources: To find the original studies that are suitable for answering the questions of this study, CNKI, VIP, WANFANG, CBM, PubMed, and EMBASE databases were searched.

Main outcome(s): Visual analogue scale (VAS ) score, Oswestry disability index (ODI ) score, and health survey summary table ( SF-36) score of leg pain and low back pain. Safety index: adverse reaction rate.

Quality assessment / Risk of bias analysis: Two researchers will use the AMSTAR2 tool to evaluate the quality of the included studies in duplicate.

Strategy of data synthesis: The results will be was briefly organized into a tabular format and analyzed using a narrative description. The data extracted from the study is presented in the form of tables and pictures. The data will be synthesized narratively and graphically.

Subgroup analysis: As scoping review research, there will be no plan for analyzing subgroup data.

Sensitivity analysis: As scoping review research, there will be no plan to perform the sensitivity analysis of data.

Country(ies) involved: China.

Keywords: Acupuncture, sciatica, scoping review, protocol.

Contributions of each author:

Author 1 - Yanji Zhang.

Email: zhangyanji1995@stmail.hbtcm.edu.cn

Author 2 - Fangyuan Liang.

Email: 1160918924@qq.com

Author 3 - Suzhen Liu.

Email: 1093494011@qq.com

Author 4 - Yingrong Zhang.

Email: 1176906801@qq.com

Author 5 - Jia Li.

Email: Ijlijia@163.com 